\title{
Existence of Solutions for a Baby-Skyrme Model
}

\author{
Hongan $\mathrm{Hu}^{1}$ and Kunlin $\mathrm{Hu}^{2}$ \\ ${ }^{1}$ Department of Mathematics, Zhoukou Normal University, Zhoukou 466000, China \\ ${ }^{2}$ College of Mathematics and Information Science, Henan University, Kaifeng 475001, China
}

Correspondence should be addressed to Kunlin Hu; hukunling1987@163.com

Received 11 April 2014; Accepted 2 July 2014; Published 15 July 2014

Academic Editor: Senlin Guo

Copyright (C) $2014 \mathrm{H}$. Hu and K. Hu. This is an open access article distributed under the Creative Commons Attribution License, which permits unrestricted use, distribution, and reproduction in any medium, provided the original work is properly cited.

The existence of the energy-minimizing solutions for a baby-Skyrme model on the sphere is proved using variational method. Some properties of the solutions are also established.

\section{Introduction}

Half a century ago, Skyrme [1] firstly suggested that the soliton in the nonlinear $\sigma$-model [2] may be explained by the baryon number, which is corresponding to the winding number of soliton.

The Skyrmions were originally introduced to describe baryons in three spatial dimensions [1]. In a nonlinear scalar field theory, a Skyrmion is a classical static field configuration of minimal energy. The scalar field is the pion field, and the Skyrmion represents a baryon. The Skyrmion has a topological charge which prevents continuously deforming to the vacuum field configuration. This charge is identified with the conserved baryon number which prevents a baryon from decaying into pions $[1,3]$.

Skyrmions have been shown to exist for a very wide class of geometries [4], which are now playing an increasing role in other areas of physics as well. For example, in certain condense matter systems, Skyrmions are used to model the bubbles that appear in the presence of an external magnetic field in two dimensions; they could provide a mechanism associated with the disappearance of antiferromagnetism, the onset of HTc superconductivity, and so on. In condensed matter physics [5], the model [6] has direct applications which may give an effective description in quantum Hall systems. In the context of condensed matter physics [7, 8 ], direct experimental observations can be made. In three spatial dimensions [6], baby Skyrmions have been studied in the context of strong interactions as a toy-model in order to understand the more complicated dynamics of usual Skyrmions which live.

In the present paper we consider a baby-Skyrme model, that is, Skyrmional model in two spatial dimensions, which was introduced in [9]. Our purpose of this paper is to establish the existence of the energy-minimizing solutions for this baby-Skyrme model rigorously by the variational method. In Section 2, we will present the mathematical structure of the model and the main existence theorem. In Section 3, we will show the existence of the energyminimizing solutions by the variational method and establish some properties of the solutions.

\section{The Mathematical Structure and Existence Theorem}

Baby Skyrmions are obtained as the nontrivial solutions of the well-known nonlinear $O(3)$ model. The model consists of three real scalars $\phi_{a}(a=1,2,3)$ subject to the constraint

$$
\vec{\phi} \cdot \vec{\phi}=1
$$

The equation of motion admits solutions with finite energy which represents a mapping of $\mathbf{R}_{\text {spat }}^{2}$ into $\mathbf{S}_{\text {int }}^{2}$. They are characterized by the density $\rho$,

$$
\rho \equiv \epsilon_{i j} \vec{\phi} \cdot\left(\partial_{i} \vec{\phi} \times \partial^{i} \vec{\phi}\right)
$$


and the winding number $W$,

$$
W=\frac{1}{8 \pi} \int d^{2} r \rho .
$$

The energy functional of this model is as follows:

$$
E=E^{(2)}+E^{(4)}+E^{(p)}
$$

with

$$
\begin{gathered}
E^{(2)}=\frac{1}{2} \int \partial_{i} \vec{\phi} \partial^{i} \vec{\phi} d^{2} r, \\
E^{(4)}=\frac{1}{8} \int \rho \rho d^{2} r, \\
E^{(p)}=\frac{\alpha}{2} \int\left(\widehat{n}_{3}-\vec{\phi}\right)^{2} d^{2} r,
\end{gathered}
$$

where $\widehat{n}_{3}$ is a unit vector in the third derivation in internal space and $\alpha$ is a parameter that is assumed positive.

By using the inequality

$$
\begin{aligned}
0 \leq \int\{ & \frac{1}{4}\left(\partial_{i} \vec{\phi} \pm \epsilon_{i j} \vec{\phi} \times \partial^{j} \vec{\phi}\right)^{2} \\
& \left.+\frac{1}{2}\left(\frac{1}{2} \epsilon_{i j} \partial^{i} \vec{\phi} \times \partial^{j} \vec{\phi} \pm \sqrt{\alpha}\left(\widehat{n}_{3}-\vec{\phi}\right)\right)^{2}\right\} d^{2} r,
\end{aligned}
$$

we may find the Bogomol'nyi bound

$$
E \geq 4 \pi k(1+\sqrt{\alpha})
$$

We are to extend the model above by going from $\mathbf{R}_{\mathrm{sp}}^{2}$ to $\mathbf{S}_{\mathrm{sp}}^{2}(L)$ where $L$ is the radius of the two-sphere. By the polar coordinates $\theta, \varphi(0 \leq \theta \leq \pi$ and $0 \leq \varphi \leq 2 \pi)$,

$$
x=L \sin \theta \cos \varphi ; \quad y=L \sin \theta \sin \varphi .
$$

And the Jacobian of the transformation and the metric associated with the polar coordinates are

$$
\begin{gathered}
J=-L^{2} \sin \theta, \\
d s^{2}=L^{2}\left(\sin ^{2} \theta d \varphi^{2}+d \theta^{2}\right) .
\end{gathered}
$$

In order to obtain explicit static solutions in the winding number $W=k$ sector, we introduce the hedgehog parameterization

$$
\begin{gathered}
\phi_{1}=\sin f \cos k \varphi ; \\
\phi_{2}=\sin f \sin k \varphi ; \\
\phi_{3}=\cos f,
\end{gathered}
$$

where

$$
f=f(\theta),
$$

is subject to the boundary conditions

$$
f(0)=\pi, \quad f(\pi)=0 .
$$

The energy functional is as follows:

$$
\begin{aligned}
& E_{k}(f) \\
& =\frac{1}{4 k} \int_{0}^{\pi} d \theta \sin \theta\left\{f^{\prime 2}+k^{2}\left(\frac{\sin f}{\sin \theta}\right)^{2}+\frac{k^{2}}{L^{2}} f^{\prime 2}\left(\frac{\sin f}{\sin \theta}\right)^{2}\right. \\
& \left.+2 \alpha L^{2}(1-\cos f)\right\},
\end{aligned}
$$

while the winding number density results in

$$
\rho_{k}=-\frac{2 k}{L^{2}} f^{\prime} \frac{\sin f}{\sin \theta} .
$$

It is not difficult to show that the Euler-Lagrange equation of (13) is

$$
\begin{aligned}
{[1+} & \left.\frac{k^{2}}{L^{2}}\left(\frac{\sin f}{\sin \theta}\right)^{2}\right] f^{\prime \prime} \\
& +\left[f^{\prime}-k^{2} \frac{\sin 2 f}{\sin 2 \theta}+\frac{k^{2}}{L^{2}} f^{\prime} \frac{\sin f}{\sin \theta}\left(f^{\prime} \frac{\cos f}{\cos \theta}-\frac{\sin f}{\sin \theta}\right)\right] \\
& \times \cot \theta-\alpha L^{2} \sin f=0 .
\end{aligned}
$$

Next we are to find a solution of the boundary problem (15) and (12). We will establish the existence of solutions by the indirect variational method.

Here is our main existence theorem, which solves the above problem.

Theorem 1. The boundary value problem (15) and (12) has a solution $f(\theta)$ such that

$$
0<f(\theta)<\pi, \quad \forall \theta \in(0, \pi),
$$

and there hold the sharp asymptotic estimates

$$
\begin{gathered}
\pi-f(\theta)=O\left(\theta^{3 / 2}\right) \quad(\text { as } \theta \longrightarrow 0), \\
f(\theta)=O\left((\pi-\theta)^{3 / 2}\right) \quad(\text { as } \theta \longrightarrow \pi) .
\end{gathered}
$$

\section{The Proof of Theorem 1}

In this section, we will divide the proof of Theorem 1 into two lemmas.

Lemma 2. The boundary value problem (15) and (12) has a solution $f(\theta)$ such that

$$
\begin{gathered}
\pi-f(\theta)=O\left(\theta^{3 / 2}\right) \quad(\text { as } \theta \longrightarrow 0), \\
f(\theta)=O\left((\pi-\theta)^{3 / 2}\right) \quad(\text { as } \theta \longrightarrow \pi) .
\end{gathered}
$$

Proof. In order to get a solution of (15) with the boundary condition (12), we may look for the minimizers of the functional (13). 
We first introduce the admissible space

$\mathscr{A}=\{f \mid f(\theta)$ is continuous on $[0, \pi]$

and absolutely continuous on every compact

subinterval of $[0, \pi]$ such that it satisfies

the boundary condition $(12)$ and $E_{k}(f)<\infty$.

Obviously the set $\mathscr{A}$ is not empty.

We intend to find a solution of (15) and (12) by solving the minimization problem:

$$
\eta \equiv \min \left\{E_{k}(f) \mid f \in \mathscr{A}\right\} .
$$

Let $\left\{f_{n}(\theta)\right\}$ be a minimizing sequence of (20). Without loss of generality, we may assume that

$$
0 \leq f_{n}(\theta) \leq \pi, \quad 0<\theta<\pi .
$$

Otherwise, we may modify the sequence to fulfill (21) meanwhile without enlarging the energy. From the inequality

$$
\begin{aligned}
\mid 1 & +\cos f_{n}(\theta) \mid \\
& \leq \int_{0}^{\theta}\left|\left(-\sin f_{n}(s)\right) f_{n}^{\prime}(s)\right| d s \\
& \leq \int_{0}^{\theta}|\sin s| \cdot \frac{\left|\sin f_{n}(s) \cdot f_{n}^{\prime}(s)\right|}{|\sin s|} d s \\
& \leq\left(\int_{0}^{\theta}|\sin s|^{2} d s\right)^{1 / 2}\left(\int_{0}^{\theta}\left(f_{n}^{\prime}(s)\right)^{2}\left(\frac{\sin f_{n}(s)}{\sin s}\right)^{2} d s\right)^{1 / 2} \\
& \leq \frac{L}{\sqrt{3} k} \theta^{3 / 2}\left(E_{k}\left(f_{n}\right)\right)^{1 / 2} \\
& =C_{1} \theta^{3 / 2}, \quad C_{1}>0,
\end{aligned}
$$

we may see that $f_{n}(\theta) \rightarrow \pi$ uniformly as $\theta \rightarrow 0$.

Similarly, we have

$$
\begin{aligned}
\mid 1 & -\cos f_{n}(\theta) \mid \\
& \leq \int_{\theta}^{\pi}\left|\sin f_{n}(s) f_{n}^{\prime}(s)\right| d s \\
& \leq \int_{\theta}^{\pi}|\sin s| \cdot \frac{\left|\sin f_{n}(s) \cdot f_{n}^{\prime}(s)\right|}{|\sin s|} d s \\
& \leq\left(\int_{0}^{\theta}|\sin s|^{2} d s\right)^{1 / 2}\left(\int_{0}^{\theta}\left(f_{n}^{\prime}(s)\right)^{2}\left(\frac{\sin f_{n}(s)}{\sin s}\right)^{2} d s\right)^{1 / 2} \\
& \leq \frac{L}{\sqrt{3} k}(\pi-\theta)^{3 / 2}\left(E_{k}\left(f_{n}\right)\right)^{1 / 2} \\
& =C_{2}(\pi-\theta)^{3 / 2}, \quad C_{2}>0 .
\end{aligned}
$$

Then, we may find that $f_{n}(\theta) \rightarrow 0$ uniformly as $\theta \rightarrow \pi$.
In view of (22) and (23), letting $n \rightarrow \infty$, we have

$$
\begin{gathered}
f_{n}(\theta) \longrightarrow \pi \quad(\text { as } \theta \longrightarrow 0), \\
f_{n}(\theta) \longrightarrow 0 \quad(\text { as } \theta \longrightarrow \pi), \\
\pi-f(\theta)=O\left(\theta^{3 / 2}\right) \quad(\text { as } \theta \longrightarrow 0), \\
f(\theta)=O\left((\pi-\theta)^{3 / 2}\right) \quad(\text { as } \theta \longrightarrow \pi) .
\end{gathered}
$$

We may get that the sequence $\left\{f_{n}(\theta)\right\}$ is bounded in $W^{1,2}(a, b)$ for any

$$
0<a<b<\pi
$$

Using weak compactness, we may assume that $\left\{f_{n}(\theta)\right\}$ (in fact, a subsequence in it) is weakly convergent in $W^{1,2}(a, b)$. Applying a diagonal subsequence argument, we may assume there is an

$$
f(\theta) \in W_{\mathrm{loc}}^{1,2}(o, \pi)
$$

such that

$$
f_{n}(\theta) \longrightarrow f(\theta) \text { as } n \longrightarrow \infty,
$$

weakly in $W^{1,2}(a, b)$. In view of the compact embedding theorem, we may get

$$
W^{1,2}(a, b) \hookrightarrow C[a, b] .
$$

That is, $W^{1,2}(a, b)$ can be compactly embedded into $C[a, b]$. So we see that the convergence $(27)$ is strong in $C[a, b]$. Consequently, we know that $f_{n}(\theta)$ is absolutely continuous in any compact subinterval of $(a, b)$ and continuous on $(0, \pi)$.

Let

$$
\begin{gathered}
F=\frac{1}{4 k} \sin \theta\left[f^{\prime 2}+k^{2}\left(\frac{\sin f}{\sin \theta}\right)^{2}+\frac{k^{2}}{L^{2}} f^{\prime 2}\left(\frac{\sin f}{\sin \theta}\right)^{2}\right. \\
\left.+2 \alpha L^{2}(1-\cos f)\right] .
\end{gathered}
$$

Using the weak lower semicontinuity property of the functional, we obtain the inequality

$$
\int_{a}^{b} F(f(\theta)) \leq \liminf _{n \rightarrow \infty} \int_{a}^{b} F\left(f_{n}(\theta)\right) d \theta \leq \liminf _{n \rightarrow \infty} E_{k}\left(f_{n}\right)=\eta,
$$

for any

$$
0<a<b<\pi
$$

Letting

$$
a \longrightarrow 0^{+}, \quad b \longrightarrow \pi^{-},
$$

we have

$$
E(f)=\int_{0}^{\pi} F(f) d \theta \leq \eta \leq E(f) .
$$


Thus we see that $f(\theta)$ fulfills the complete boundary conditions (12). Therefore

$$
f(\theta) \in \mathscr{A},
$$

and (30) allows us to obtain

$$
E(f)=\eta
$$

That is, $f$ is found to be a solution of (20). As a consequence, $f$ is a finite-energy solution of (12) and (15).

Next we will establish some properties of the energyminimizing solutions.

Lemma 3. Let $f$ be the energy-minimizing solution obtained in Lemma 2. Then

$$
0<f(\theta)<\pi, \quad \forall \theta \in(0, \pi) .
$$

Proof. Evidently, $f(\theta) \equiv 0$ is an equilibrium point of (15). We assume that there is $\theta_{0}$ such that

$$
f\left(\theta_{0}\right)=0 .
$$

Hence, $f(\theta)$ attains its global minimum, so

$$
f^{\prime}\left(\theta_{0}\right)=0 .
$$

Using the uniqueness theorem for the initial value problem of ordinary differential equations, we can get

$$
f(\theta) \equiv 0,
$$

which contradicts

$$
f(0)=\pi
$$

so

$$
f(\theta)>0, \quad \forall \theta \in(0, \pi) .
$$

Similarly, we may find that

$$
f(\theta)<\pi, \quad \forall \theta \in(0, \pi) .
$$

Combining Lemmas 2 and 3, we complete the proof of Theorem 1.

\section{Conflict of Interests}

The authors declare that there is no conflict of interests regarding the publication of this paper.

\section{Acknowledgments}

This work was supported by the Natural Science Foundation of Henan Province under Grant 122300410188. The authors thank the referees for their valuable suggestions which improve the quality of this paper. They also thank Professor S.-X. Chen for his guidance and assistance throughout the work.

\section{References}

[1] T. H. R. Skyrme, "A non-linear field theory," Proceedings of the Royal Society A: Mathematical, Physical and Engineering Sciences, vol. 260, pp. 127-138, 1961.

[2] Y. Yang, Solitons in Field Theory and Nonlinear Analysis, Springer, New York, NY, USA, 2001.

[3] T. H. R. Skyrme, "A unified field theory of mesons and baryons," Nuclear Physics, vol. 31, pp. 556-569, 1962.

[4] N. S. Manton, "Geometry of skyrmions," Communications in Mathematical Physics, vol. 111, no. 3, pp. 469-478, 1987.

[5] A. D. Jackson, N. S. Manton, and A. Wirzba, "New skyrmion solutions on a 3-sphere," Nuclear Physics A, vol. 495, no. 3-4, pp. 499-522, 1989.

[6] B. M. A. Piette, B. J. Schroers, and W. J. Zakrzewski, "Dynamics of baby skyrmions," Nuclear Physics B, vol. 439, no. 1-2, pp. 205235, 1995

[7] S. L. Sondhi, A. Karlhede, S. A. Kivelson, and E. H. Rezayi, "Skyrmions and the crossover from the integer to fractional quantum Hall effect at small Zeeman energies," Physical Review B, vol. 47, no. 24, pp. 16419-16426, 1993.

[8] X. Z. Yu, Y. Onose, N. Kanazawa et al., "Real-space observation of a two-dimensional skyrmion crystal," Nature, vol. 465, no. 7300, pp. 901-904, 2010.

[9] N. N. Scoccola and D. R. Bes, "Two-dimensional skyrmions on the sphere," Journal of High Energy Physics, vol. 1998, no. 9, 9 pages, 1998. 


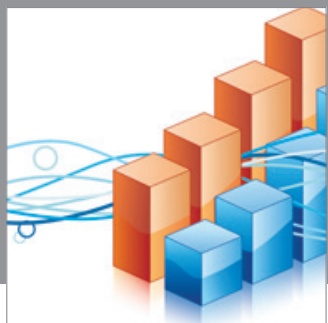

Advances in

Operations Research

mansans

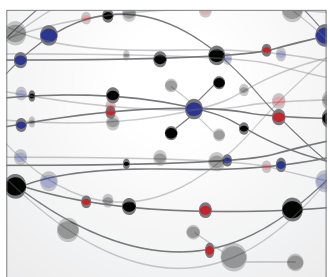

The Scientific World Journal
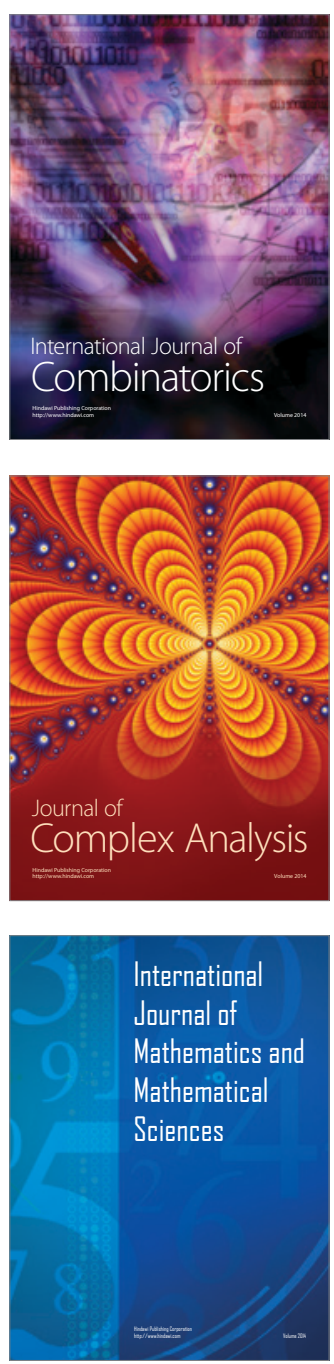
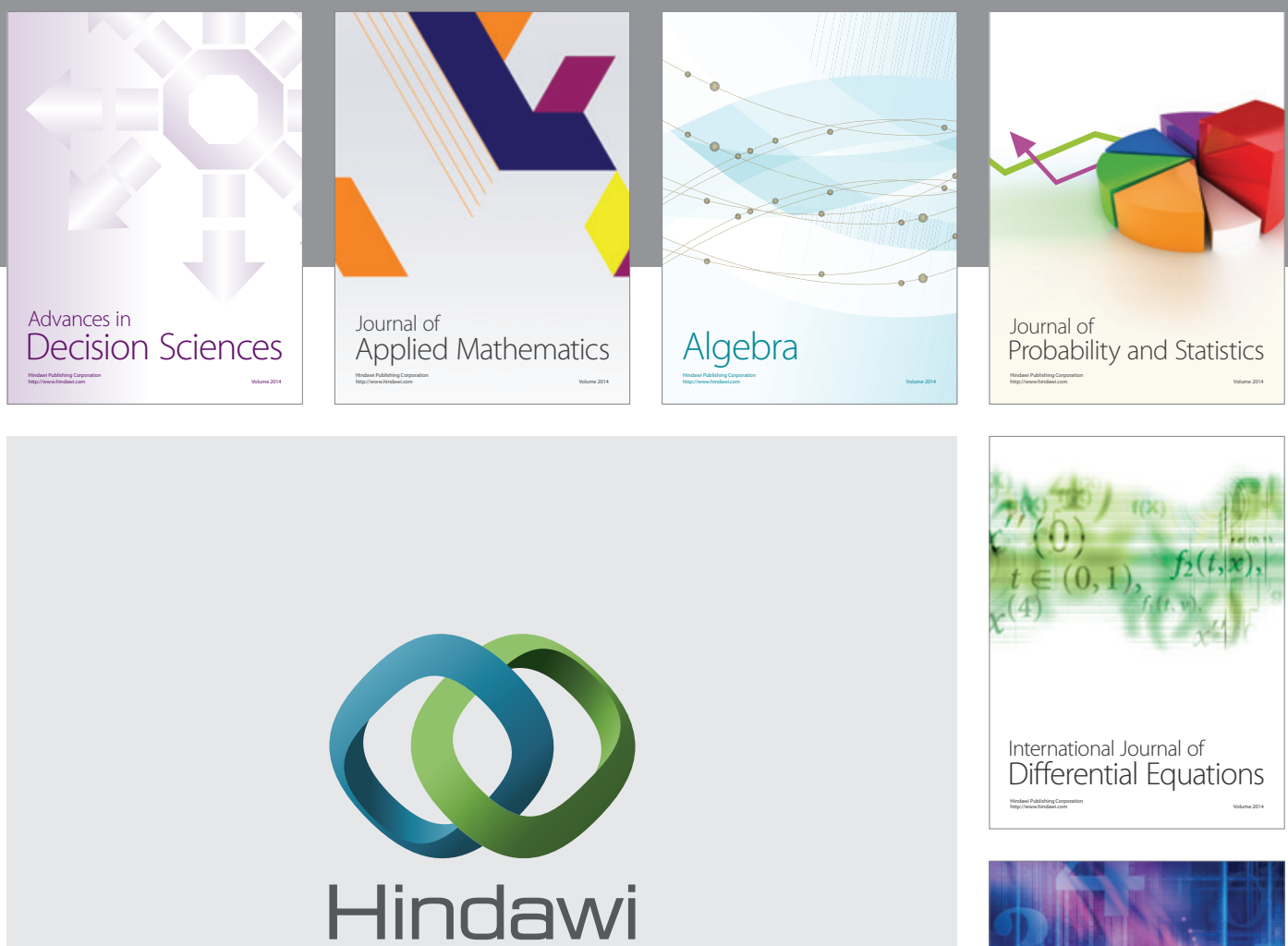

Submit your manuscripts at http://www.hindawi.com
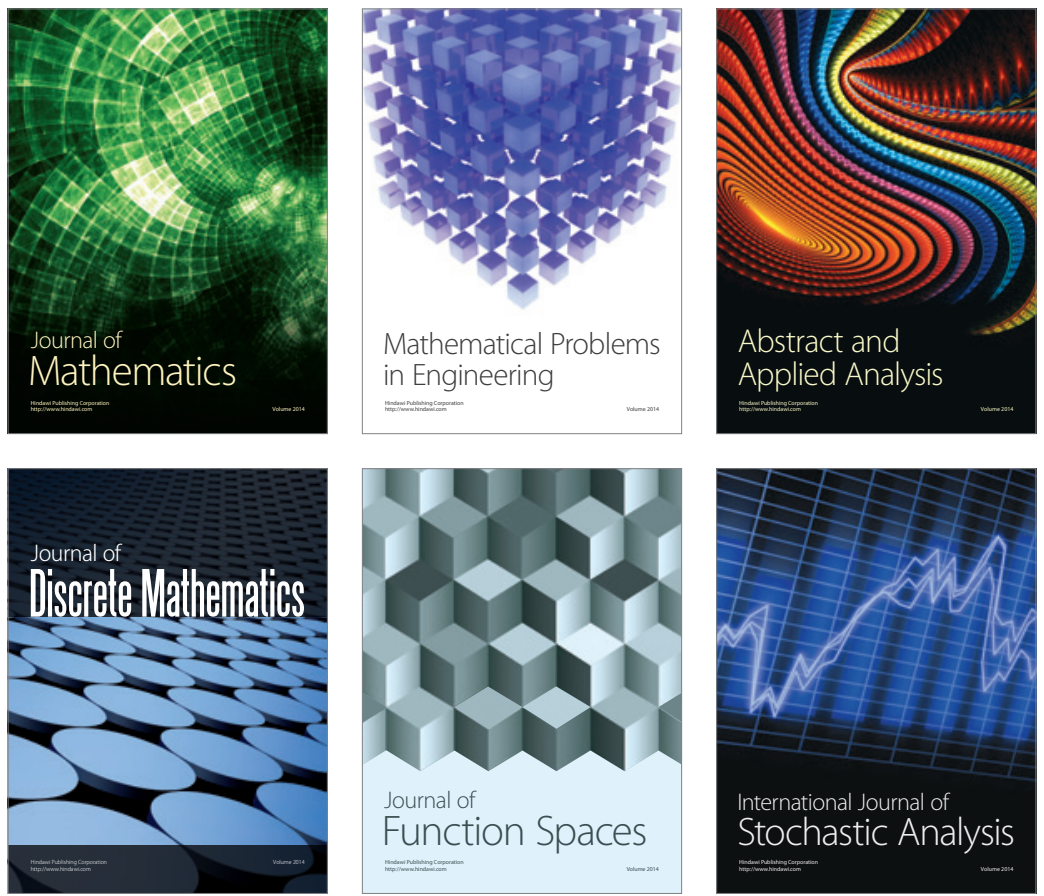

Journal of

Function Spaces

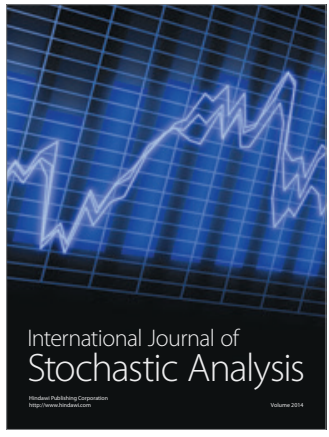

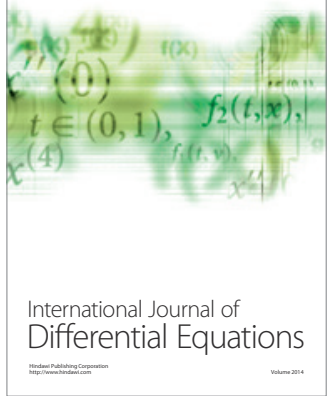
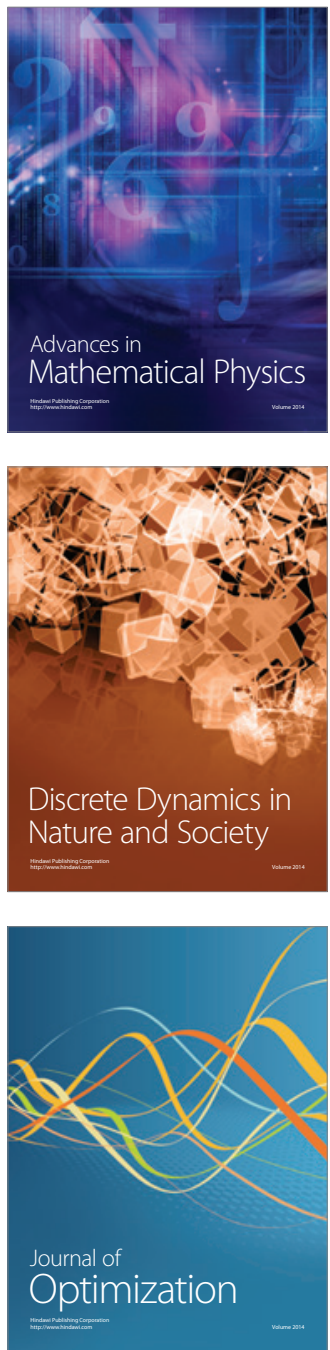